\title{
Für Kinder noch keine Daten
}

\author{
Erwachsene Patienten mit besonders schweren anaphylaktischen \\ Reaktionen nach Insektenstichen weisen gehäuft eine Erhöhung der \\ basalen Serumtryptasekonzentration auf. Ein Leser fragt nach zum \\ praktischen Vorgehen bei Kindern mit Mastzellerkrankung.
}

m Hinblick auf die Leitlinie „Erhöhte basale Serumtryptasekonzentration oder Mastozytose als Risikofaktor der Hymenopterengiftallergie" (Allergo J 2004; 13: 440-2) habe ich folgende Fragen: Ich betreue zwei Kinder mit einer kutanen Mastozytose, bei denen es bisher zu keinen Allgemeinreaktionen auf Insektenstiche gekommen ist. Sollte man bei ihnen „einfach so" die Insektengift-

\section{Stellungnahme}

D ie genannte Leitlinie bezieht sich auf erwachsene Patienten, für Kinder liegen bisher keine Daten vor.

Die Indikation zur Insektengifthyposensibilisierung wird bei Patienten mit erhöhter basaler Serumtryptasekonzentration oder Mastozytose (zusammenfassend „Mastzellerkrankung“") nach derzeitigem Kenntnisstand bisher nicht anders als bei solchen ohne Hinweise auf diese Begleiterkrankung gestellt, d.h. ohne Vorgeschichte einer systemischen Reaktion vom Soforttyp wird nicht hyposensibilisiert. Diagnostik zum Nachweis einer Sensibilisierung ist also nicht empfehlenswert, da sich hieraus keine therapeutischen Konsequenzen ergeben und bei positivem Testausfall mit einer erheblichen Verunsicherung von Arzt und Patient zu rechnen ist. Sicherheitshalber würden wir jedoch dazu raten, Kinder mit Mastzellerkrankung und systemischer Soforttypreaktion auf einen Insektenstich auch dann zu hyposensibilisieren, wenn sie nur eine systemische Hautreaktion (generalisierte Urtikaria, Angioödem ohne Bezug zur Stichstelle, Flush, generalisierter Juckreiz) erlitten haben - also eine Reaktion, bei der nach aktuellem Kenntnisstand bei Kindern ansonsten eine Hyposensibilisierung meist nicht spezifischen IgE-Antikörper im Serum bestimmen und Hauttests mit Insektengiften vornehmen? Gibt es Erkenntnisse darüber, ob eine erhöhte basale Serumtryptasekonzentration bei diesen Kindern auf ein erhöhtes Risiko für systemische Reaktionen hinweist?

Andreas Schlossbauer Facharzt für Kinder und Jugendheilkunde Bad Kissingen

nötig ist. Dies ist eine Vorsichtsmaßnahme, Untersuchungen hierzu gibt es bisher nicht.

Ob Patienten mit Mastzellerkrankung ein erhöhtes Risiko für das Auftreten von systemischen Insektenstichreaktionen haben, ist bisher nicht bekannt. Wir wissen nur, dass bei solchen Patienten auftretende Reaktionen sehr oft besonders schwer verlaufen. Sollte sich in Zukunft zeigen, dass Mastzellerkrankung auch ein Risiko für das Auftreten einer Insektengiftallergie ist, müsste die Frage nach einer „prophylaktischen“ Insektengifthyposensibilisierung neu diskutiert werden.

Bei Patienten mit Mastozytose sollte die Serumtryptasekonzentration im Rahmen der Diagnostik zur Erfassung der Krankheitsausdehnung bestimmt werden. Hinsichtlich der Insektengiftallergie ergeben sich aus der Serumtryptasekonzentration bei bestehender Mastozytose keine neuen Konsequenzen, da das Risiko durch die erhöhte basale Serumtryptasekonzentration oder die Mastozytose begründet wird.

Prof. Dr. Bernhard Przybilla Dr. Franziska Ruëff Klinik und Poliklinik für Dermatologie und Allergologie

Ludwig-Maximilians-Universität Frauenlobstraße 9-11 80337 München 